\title{
Numerical Solution of Discrete SIR Model with Horizontal and Vertical Transmission
}

\author{
Trija Fayeldi \\ Universitas Kanjuruhan Malang \\ Email: trija_fayeldi@unikama.ac.id
}

\begin{abstract}
The aim of this paper is to is to generalize the discrete model with horizontal and vertical transmission. We use Euler method to approximate numerical solution of the model. We found two equilibrium points, that aredisease free and endemic equilibrium points. The existence of these points depend on basic reproduction number $R_{0}$ and step size $h$. We found that if $R_{0}<1$ then only disease free equilibrium points exists, while both points exists when $R_{0}>1$. We also found that the stability of these equilibrium points depend on the value of step-size $h$. Some numerical experiments were presented as illustration of the discussion result.
\end{abstract}

Keywords: discrete model; numerical Solution; transmission; stability

\section{INTRODUCTION}

Recently, disease model has been studied widely by many authors [1], [2], [3]. In [1] the authors develop and anlyse two population-based model of the transmission dynamics of the worm parasite while in [2] the authors introduced an IDEA (incidence decay with exponential adjustment) model that can be used for short-term epidemiological forecasting. The exact solution of SIR model an SVIR epidemic model with deadly deseases has been investigated by [3]. One tool to analyze the spreading of a disease is mathematical models. The common procedure in modelling disease spreading is by using compartment model. In compartment model, the population is divided into two different classes, that is susceptible and infected, called the SI model. If we assume that some of the infected individual will recover then we include recovered class called as SIR model.

The exact solution of many disease model can not be solved easily due to the complexity of the model. In this case, the numerical approach will do some help in finding the solution [4]. One of the useful numerical method that we can use is Euler method. This method give a good approximation in many works, such as in [5] and [6]. In [5] the method was applied to approximate SIRS epidemic model while in [6] it was applied to find the numerical solution of SIR Pairwise Epidemic model. Euler method is a simple scheme, but only works for a small step-size. In this paper, we use Euler method to develop discrete version of the model and analyze the stability around its equilibrium points. We use stepsize $h$ as main parameter to determine the stability of those equilibrium points. Finally, we present stability of the each equilibrium points. 
The organization of this paper is as follows. In Method section, we develop the discrete version of SIR disease model with vertical and horizontal transmission compartment model. In Results and Discussion section, we present sufficient conditions for stability of the disease free equilibrium and endemic equilibrium, followed by some numerical simulation to illustrate our theoretical finding. Finally, we conclude our result in the last section.

\section{METHODS}

The method we use in this paper is literature study. The model we use in this paper was proposed by [7] as follow.

$$
\begin{aligned}
& \frac{d S}{d t}=-\beta S I-b S-(b-p d) I+b \\
& \frac{d I}{d t}=\beta S I-p d \mathrm{I}-r \mathrm{I}
\end{aligned}
$$

where $S(t)$ and $I(t)$ denote the number of the susceptible and infective individual respectively. The susceptible become infectious at rate $\beta I$, where $\beta$ is the contact rate; while $b$ represent birth rate and $d$ represent death rate. The infectious recover at the rate $r$; while $p$ is the proportion of infective parents offspring that are susceptible individuals. All parameters are assumed to be positive. We assume that $b=d$ and $\quad 0<p<1$ as in [7]. Hence, the condition $b-p d>0$ must hold. The basic reproduction number of model (1) is $R_{0}=\frac{\beta}{p d+\mathrm{r}}$. Transfer diagram of model (1) can be seen in Figure 1.

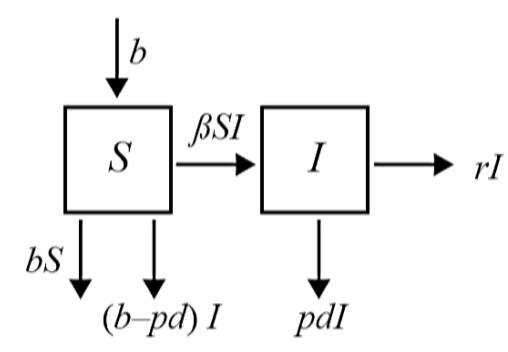

Figure 1. Transfer Diagram of the Model

Next, we use Euler method to discretize the model and use h as step-size. We have $\frac{S_{n+1}-S_{n}}{h}=-\beta S_{n} I_{n}-b S_{n}-(b-p d) I_{n}+b$

$\frac{I_{n+1}-I_{n}}{h}=\beta S_{n} I_{n}-p d I_{n}-r I_{n}$

We can rewrite (2) as

$$
\begin{aligned}
& S_{n+1}=S_{n}+h\left(-\beta S_{n} I_{n}-b S_{n}-(b-p d) I_{n}+b\right) \\
& I_{n+1}=I_{n}+h\left(\beta S_{n} I_{n}-(p d+r) I_{n}\right)
\end{aligned}
$$




\section{Theorem 1}

There exists two equilibrium points of model (3), that is $E_{0}=(1,0)$ and $E_{\mathrm{e}}=$ $\left(\frac{1}{R_{0}}, \frac{b\left(R_{0}-1\right)}{\beta+(b-p d) R_{0}}\right)$.

\section{Proof:}

Let $S^{*}$ dan $I^{*}$ are equilibrium point of model (3), then we have

$S^{*} \quad=S^{*}+h\left(-\beta S^{*} I^{*}-b S^{*}-(b-p d) I^{*}+b\right)$

$I^{*} \quad=I^{*}+h\left(\beta S^{*} I^{*}-(p d+r) I^{*}\right)$

From equation (5), we have

$0 \quad=h\left(\beta S^{*} I^{*}-(p d+r) I^{*}\right)$

Since $h>0$, we can conclude that

$0 \quad=\beta S^{*} I^{*}-(p d+r) I^{*}$

Thus, $I^{*}=0$

$$
=I^{*}\left(\beta S^{*}-(p d+r)\right)
$$

or

$\beta S^{*}-(p d+r)=0$

Subtituting (6) to (4) leads to $S^{*}=1$. So, the disease free equilibrium point is $E_{0}=(1,0)$.

From (7), we have $S^{*}=\frac{p d+r}{\beta}=\frac{1}{R_{0}}$

Subtituting (8) to (4) leads to $I^{*}=\frac{b\left(R_{0}-1\right)}{\beta+(b-p d) R_{0}}$. So, the endemic equilibrium point is $E e=\left(\frac{1}{R_{0}}, \frac{b\left(R_{0}-1\right)}{\beta+(b-p d) R_{0}}\right)$. Notice that $I^{*}=\frac{b\left(R_{0}-1\right)}{\beta+(b-p d) R_{0}}>0$ when $R_{0}-1>0$, in other word $R_{0}>1$.

\section{RESULTS AND DISCUSSION}

In this section, we will discuss about stability of the model around those two equilibrium points and other phenomenon that occured. Let us rewrite equation (3) as follow.

$F(S, I)=(1-h b) S-h \beta S I-h(b-p d) I+h b$

$G(S, I)=I+h \beta S I-h(p d+r) I$

Jacobian matrix of equation (3) can be written as follow.

$J=\left(\begin{array}{ll}\frac{\partial F}{\partial S} & \frac{\partial F}{\partial I} \\ \frac{\partial G}{\partial S} & \frac{\partial G}{\partial I}\end{array}\right)$

where $\frac{\partial F}{\partial S}=1-h(b+\beta I) ; \frac{\partial F}{\partial I}=-h(\beta S+(b-p d)) ; \quad \frac{\partial G}{\partial S}=h \beta I ; \quad \frac{\partial G}{\partial S}=1+h(\beta S-(p d+r))$.

Now, we can analyze the stability of equilibrium points using matrix (9). 


\section{Lemma 2}

If $R_{0}<1$ and $h<\min \left\{\frac{2}{b}, \frac{2}{(p d+r)\left(1-R_{0}\right)}\right\}$ then $E_{0}$ is locally asymptotically stable.

\section{Proof:}

Since $R_{0}=\frac{\beta}{p d+r}$, we have $1-R_{0}=1-\frac{\beta}{p d+r}=\frac{(p d+r)-\beta}{p d+r}$. Hence,

$(p d+r)-\beta=(p d+r)\left(1-R_{0}\right)$. Clearly, $(p d+r)-\beta>0$ if $R_{0}<1$. Subtituting $E_{0}=(1,0)$ to matrix (9) lead us to

$$
J_{E_{0}}=\left(\begin{array}{cc}
1-h b & -h(\beta+(b-p d)) \\
0 & 1-h((p d+r)-\beta)
\end{array}\right)=\left(\begin{array}{cc}
1-h b & -h(\beta+(b-p d)) \\
0 & 1-h(p d+r)\left(1-R_{0}\right)
\end{array}\right)
$$

It is easy to find that the eigenvalues of matrix (10) are $\lambda_{1}=1-h b$ and $\lambda_{2}=1-h(p d+r)$ $\left(1-R_{0}\right)$. If $\mathrm{h}<\frac{2}{b}$ then we have $-1<1-h b<1$ which lead us to $\left|\lambda_{1}\right|=|1-h b|<1$. In other hand, if $\mathrm{h}<\frac{2}{(p d+r)\left(1-R_{0}\right)}$ then we have $-1<1-h(p d+r)\left(1-R_{0}\right)<1$, that is $\left|\lambda_{2}\right|=\mid 1-$ $h(p d+r)\left(1-R_{0}\right) \mid<1$. Hence $E_{0}$ stable if and only if $\left|\lambda_{\mathrm{i}}\right|<1, \mathrm{i}=1,2$, which is equivalent with $\mathrm{h}<\min \left\{\frac{2}{b}, \frac{2}{(p d+r)\left(1-R_{0}\right)}\right\}$.

Again, by subtituting $E_{e}$ to Jacobian matrix (9), we have $J_{E_{e}}=\left(\begin{array}{cc}1-h(b+c) & -h(b+r) \\ h c & 1\end{array}\right)$ where $c=\frac{b\left(R_{0}-1\right)(p d+r)}{(b+r)}$. Let $\tau=1-h(b+c)+1=2-h(\mathrm{~b}+\mathrm{c})$ and $\Delta=(1-h(b+c))$ (1) $-(h c)(-h(b+r))=c(b+r) h^{2}-(b+c) h+1$. Notice that since $R_{0}>1$, then $c(b+r)=b$ $\left(R_{0}-1\right)(p d+r)>0$. Now, we analyze the stability of $E_{e}$. We use the following theorem.

\section{Theorem 3}

The root of $p(\lambda)=\lambda^{2}-\tau \lambda+\Delta$ satisfy $\left|\lambda_{i}\right|<1, i=1,2$ if and only if the following conditions hold:
1. $p(1)=1-\tau+\Delta>0$
2. $p(0)=\Delta<1$
3. $p(-1)=1+\tau+\Delta>0$

We break these theorem into several lemmas as follow and apply them to model (3).

\section{Lemma 4}

If $R_{0}>1$ then $p(1)>0$.

\section{Proof:}

In model (3), it is clear that if $R_{0}>1$ then $c(b+r)>0$. Hence,

$p(1)=1-(2-h(b+c))+\left(c(b+r) h^{2}-(b+c) h+1\right)=c(b+r) h^{2}>0$ 


\section{Lemma 5}

If $R_{0}>1$ and $h<\frac{b+c}{c(b+r)}$ then $p(0)<1$.

\section{Proof:}

Suppose $R_{0}>1$, then we have $R_{0}-1>0$, so that

$\frac{b+c}{c(b+r)}=\frac{b+\left(\frac{b\left(R_{0}-1\right)(p d+r)}{(b+r)}\right)}{\left(\frac{b\left(R_{0}-1\right)(p d+r)}{(b+r)}\right)(b+r)}>0$. Thus, if $h<\frac{b+c}{c(b+r)}$ then we have

$c(b+r) h^{2}-(b+c) h+1=\Delta<1$

For the last condition, notice that $p(-1)=1+\tau+\Delta=1+(2-h(b+c))+\left(\mathrm{c}(b+r) h^{2}-(b+\right.$ c) $h+1)=c(b+r) h^{2}-2(b+c) h+4$. Let $f(h)=A h^{2}+B h+C$, where $A=c(b+r)>0, B=-$ $2(b+c)$, and $C=4$. Solutions of $f(h)=A h^{2}+B h+C=0$ is

$$
\begin{aligned}
h_{1,2} & =\frac{-B \pm \sqrt{B^{2}-4 A C}}{2 A} \\
& =\frac{-(-2(b+c)) \pm \sqrt{(-2(b+c))^{2}-4 c(b+r)(4)}}{2 c(b+r)} \\
& =\frac{(b+c)}{c(b+r)}\left(1 \pm \sqrt{1-\frac{4 c(b+r)}{(b+c)^{2}}}\right)
\end{aligned}
$$

\section{Lemma 6}

If one of the following condition is hold, they are:

1. $1-\frac{4 c(b+r)}{(b+c)^{2}}<0$; or

2. $1-\frac{4 c(b+r)}{(b+c)^{2}}=0$ and $h \neq \frac{b+c}{c(b+r)}$;or

3. $1-\frac{4 c(b+r)}{(b+c)^{2}}>0$ and $h<h_{1}$ or $h>h_{2}$

where $h_{1}=\frac{(b+c)}{c(b+r)}\left(1-\sqrt{1-\frac{4 c(b+r)}{(b+c)^{2}}}\right)$ and $h_{2}=\frac{(b+c)}{c(b+r)}\left(1+\sqrt{1-\frac{4 c(b+r)}{(b+c)^{2}}}\right)$

then $p(-1)>0$.

\section{Proof:}

1. Notice that $1-\frac{4 c(b+r)}{(b+c)^{2}}<0$ lead us to $(b+c)^{2}<4 b\left(R_{0}-1\right)(p d+r)$.

It means that $R_{0}>1+\frac{(b+c)^{2}}{4 b(p d+r)}>1$. Since $1-\frac{4 c(b+r)}{(b+c)^{2}}<0$ and $A=c(b+r)>0$ 
then $p(-1)=1+\tau+\Delta=f(h)=A h^{2}+B h+C>0$ for all $h>0$.

2. It easy to find that $1-\frac{4 c(b+r)}{(b+c)^{2}}=0$ equivalent with $R_{0}=1+\frac{(b+c)^{2}}{4 b(p d+r)}>1$.

Since $1-\frac{4 c(b+r)}{(b+c)^{2}}=0$, we have $h=\frac{(b+c)}{c(b+r)}$.

Since $A=c(b+r)>0$, if we set $h \neq \frac{(b+c)}{c(b+r)}$ then

$p(-1)=1+\tau+\Delta=f(h)=A h^{2}+B h+C>0$.

3. We know that $1-\frac{4 c(b+r)}{(b+c)^{2}}>0$ equivalent with $1<R_{0}<1+\frac{(b+c)^{2}}{4 b(p d+r)}$.

Since $1-\frac{4 c(b+r)}{(b+c)^{2}}>0$, we have $h_{1,2}=\frac{(b+c)}{c(b+r)}\left(1 \pm \sqrt{1-\frac{4 c(b+r)}{(b+c)^{2}}}\right)$.

Let $h_{1}=\frac{(b+c)}{c(b+r)}\left(1-\sqrt{1-\frac{4 c(b+r)}{(b+c)^{2}}}\right)$ and $h_{2}=\frac{(b+c)}{c(b+r)}\left(1+\sqrt{1-\frac{4 c(b+r)}{(b+c)^{2}}}\right)$.

Notice that if $R_{0}>1$ then $0<\sqrt{1-\frac{4 c(b+r)}{(b+c)^{2}}}<1$.

Thus, $1-\sqrt{1-\frac{4 c(b+r)}{(b+c)^{2}}}>0$ and $1+\sqrt{1-\frac{4 c(b+r)}{(b+c)^{2}}}>0$.

Since $0<1-\sqrt{1-\frac{4 c(b+r)}{(b+c)^{2}}}<1+\sqrt{1-\frac{4 c(b+r)}{(b+c)^{2}}}$, we can conclude that $h_{1}<h_{2}$.

We have $A=c(b+r)>0$, if we set $h<h_{1}$ or $h>h_{2}$ then

$p(-1)=1+\tau+\Delta=f(h)=A h^{2}+B h+C>0$.

(Qed)

We can generate Lemma 4, Lemma 5, and Lemma 6 into Theorem 7.

\section{Theorem 7}

If $R_{0}>1$ and one of the following condition hold:

1. $h<\frac{(b+c)}{c(b+r)}$, or

2. $h<\min \left\{\frac{(b+c)}{c(b+r)}, \frac{(b+c)}{c(b+r)}\left(1-\sqrt{1-\frac{4 c(b+r)}{(b+c)^{2}}}\right)\right\}$

then the equilibrium point $E_{e}$ is locally asymptotically stable.

We investigate the numerical solution of model (3) through some numerical experiment. We set parameters as in Table 1 below such that $R_{0}<1$. 
Table 1. Parameters for $R_{0}<1$

\begin{tabular}{cc}
\hline Parameters & Value \\
\hline$\beta$ & 0.05 \\
$b$ & 0.9 \\
$p$ & 0.8 \\
$d$ & 0.9 \\
$r$ & 0.8 \\
$h$ & 0.1 \\
\hline
\end{tabular}

From this case, we find that $R_{0}=0.0329 \leq 1,\left|\lambda_{1}\right|=0.91<1$ and $\left|\lambda_{2}\right|=0.853<1$, and $h=0.1<1.36054$. Figure 2 shows S-I plot of the model using parameters in Table 1 .

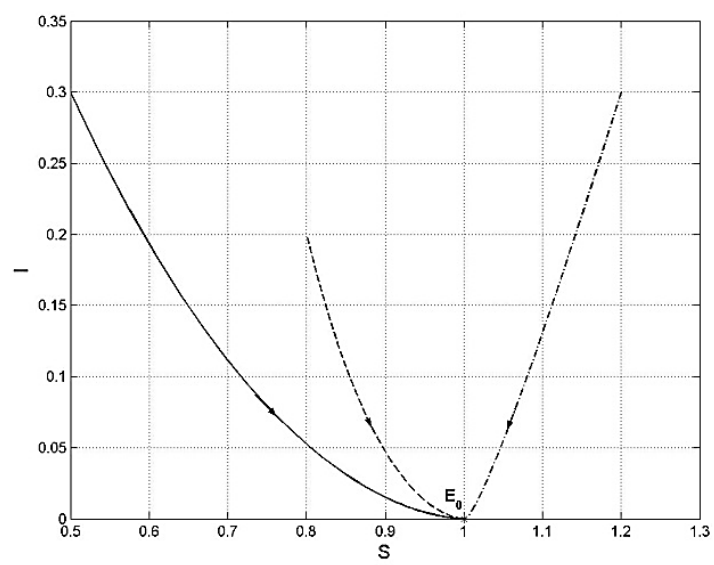

Figure 2. Plot of the model with $\beta=0.05, b=0.9, p=0.8, d=0.9, r=0.8$, and $h=0.1$

We use three different initial conditions in Figure 1. We see that all solutions goes to $E_{0}$ which means that $E_{0}$ is locally asymptotically stable for such parameters. For the next experiment, we set parameters as in Table 2.

Table 2. Parameters for $R_{0}>1$

\begin{tabular}{cc}
\hline Parameters & Value \\
\hline$\beta$ & 2 \\
$b$ & 0.7 \\
$p$ & 0.9 \\
$d$ & 0.7 \\
$r$ & 0.8 \\
$h$ & 0.1 \\
\hline
\end{tabular}

From this case, we find that $R_{0}=1.3986>1, \lambda_{1}=0.992+0.0224 i$ and $\lambda_{2}=0.992-$ $0.0224 i$, and $h=0.1<2.8655$. Figure 3 shows S-I plot of the model using parameters in Table 2. 


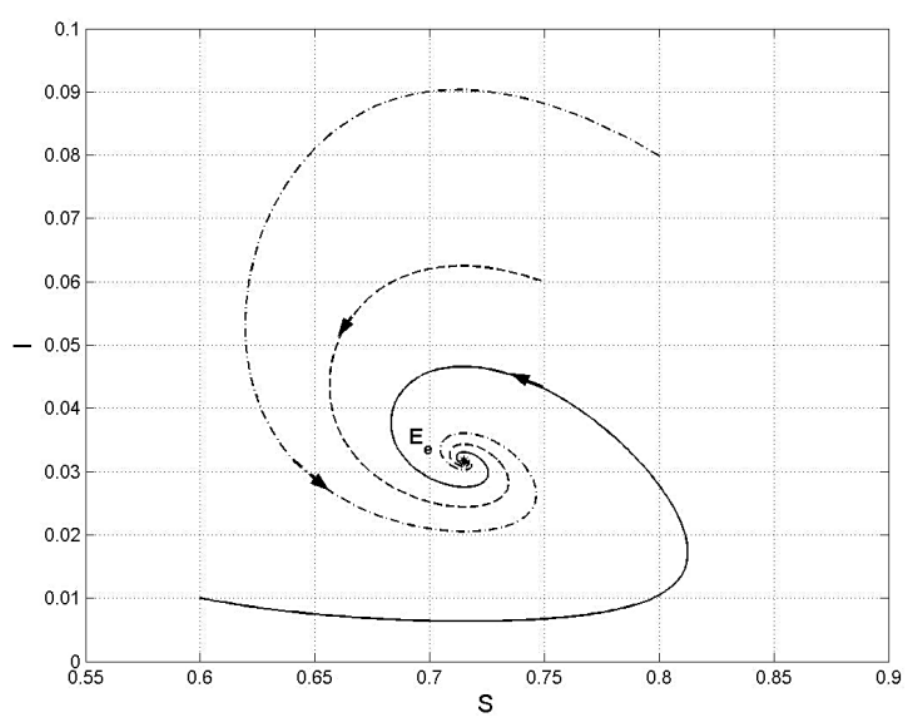

Figure 3. Plot of the model with $\beta=2, b=0.7, p=0.9, d=0.7, r=0.8$, and $h=0.1$

We use three different initial conditions in Figure 3. We see that all solutions goes to $E_{e}$ which means that $E_{e}$ is locally asymptotically stable for such parameters. For the last experiment, we set parameters as in Table 3.

Table 3. Parameters for $R_{0}>1$

\begin{tabular}{cc}
\hline Parameters & Value \\
\hline$\beta$ & 0.8 \\
$b$ & 1.2 \\
$p$ & 0.2 \\
$d$ & 1.2 \\
$r$ & 0.1 \\
$h$ & 0.1 \\
\hline
\end{tabular}

From this case, we find that $R_{0}=6.67>1,\left|\lambda_{1}\right|=0.89479<1$ and $\left|\lambda_{2}\right|=0.92244<1$, and $h=0.1<1.9$. Figure 4 shows S-I plot of the model using parameters in Table 3.

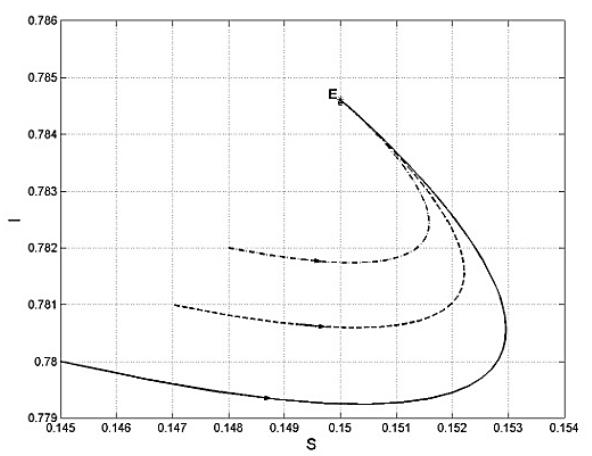

Figure 4. Plot of the model with $\beta=0.8, b=1.2, p=0.2, d=1.2, r=0.1$, and $h=0.1$ 
Again, we use three different initial conditions in Figure 4. We see that all solutions goes to $E_{e}$ which means that $E_{e}$ is locally asymptotically stable for such parameters.

\section{CONCLUSIONS}

In this paper, we have investigated a discrete numerical solution of SIR model with horizontal and vertical transmission. Euler method is use to discretisize the model. We found two equilibrium points that are $E_{0}$ and $E_{e}$. The stability of these equilibrium points are depend on a reproduction number $R_{0}$ and step size $h$. It can be shown that the discrete model is dynamically consistent with its continuous model only for relatively small stepsize $h$. We only investigate for stable case (sink). Further works is needed to investigate for the unstable cases, such as saddle and source, including bifurcation phenomenon of the model.

\section{REFERENCES}

[1] C. Bürli et al., "Mathematical analysis of the transmission dynamics of the liver fluke, opisthorchis viverrini," Journal of Theoretical Biology, 439(2018): 181-194, 2018.

[2] M. Santillana et al., "Relatedness of the incidence decay with exponential adjustment (IDEA) model farr's law and SIR compartmental difference equation models," Infectious Disease Modelling, 3(2018): 1-12, 2018.

[3] J. Harianto, and T. Suparwati, "Local stability analysis of an svir epidemic model," CAUCHY -Jurnal Matematika Murni dan Aplikasi, 5(1)(2017): 20-28, 2017.

[4] R.N. Dinnullah and T. Fayeldi, T, "A discrete numerical scheme of modified leslie-gower with harvesting model," CAUCHY -Jurnal Matematika Murni dan Aplikasi, 5(2)(2018): 42-47, 2018.

[5] D. Kiouach and Y. Sabbar, "Stability and threshold of a stochastic SIRS epidemic model with vertical transmission and transfer from infectious to susceptible individuals," Discrete Dynamics in Nature and Society, 2018: 1-13, 2018.

[6] W. Jing, Z. Jin, and J. Zhang, "An SIR pairwise epidemic model with infection age and demography," Journal of Biological Dynamics, 12(1): 486-508, 2018.

[7] X. Meng and L. Chen, "The dynamics of a new SIR epidemic model concerning pulse vaccination strategy," Applied Mathematics and Computation, 197(2008): 582597, 2008. 